\title{
Helmholtz Pulse Propagation \& Spatially-Dispersive Light
}

\author{
J. M. Christian, T. F. Hodgkinson and G. S. McDonald \\ Joule Physics Laboratory, School of Computing, Science and Engineering, \\ Materials \& Physics Research Centre, University of Salford, Salford M5 4WT, U.K. \\ P. Chamorro-Posada \\ Departmento de Teoría de la Señal y Comunicaciones e Ingeniería Telemática, Universidad de Valladolid, \\ ETSI Telecomunicación, Campus Miguel Delibes s/n, 47011 Valladolid, Spain
}

\begin{abstract}
We present the first detailed account of modelling pulses in Helmholtz-type nonlinear systems with both temporal and spatial dispersion. Exact analytical solitons will be reported, and their stability examined through mathematical analysis and computer simulations.

(C) 2010 Optical Society of America

OCIS codes: (190.5530) Pulse propagation and temporal solitons; (190.4400) Nonlinear optics, materials.
\end{abstract}

\section{Introduction}

We propose, for the first time to our knowledge, a new generic model for describing the evolution of scalar optical pulses in Kerr-type waveguides. The (normalized) wave envelope $u$ satisfies a governing equation that is of the nonlinear Helmholtz type, namely

$$
\kappa \frac{\partial^{2} u}{\partial \zeta^{2}}+i\left(\frac{\partial u}{\partial \zeta}+\alpha \frac{\partial u}{\partial \tau}\right)+\frac{s}{2} \frac{\partial^{2} u}{\partial \tau^{2}}+|u|^{2} u=0
$$

where the space and time coordinates are denoted by $\zeta$ and $\tau$, respectively, $\alpha$ is related to the group velocity, and $s=$ \pm 1 flags the anomalous/normal temporal dispersion regime. The first term in Eq. (1) embodies spatial dispersion, of which there are two main sources in semiconductor waveguides: the propagation contribution (inherent to any propagating electromagnetic mode) and a recently-proposed material contribution that arises from field-exciton coupling [1]. We will show how much headway can be made when abandoning the universal slowly-varying envelope approximation, and the subsequent Galilean boost to a local time frame. Together, these two simplifications lead to a theory of optical pulses based on the nonlinear Schrödinger (NLS) equation, with all its advantages and disadvantages. Here, we develop a more general Helmholtz formalism and uncover a broad range of new physical predictions.
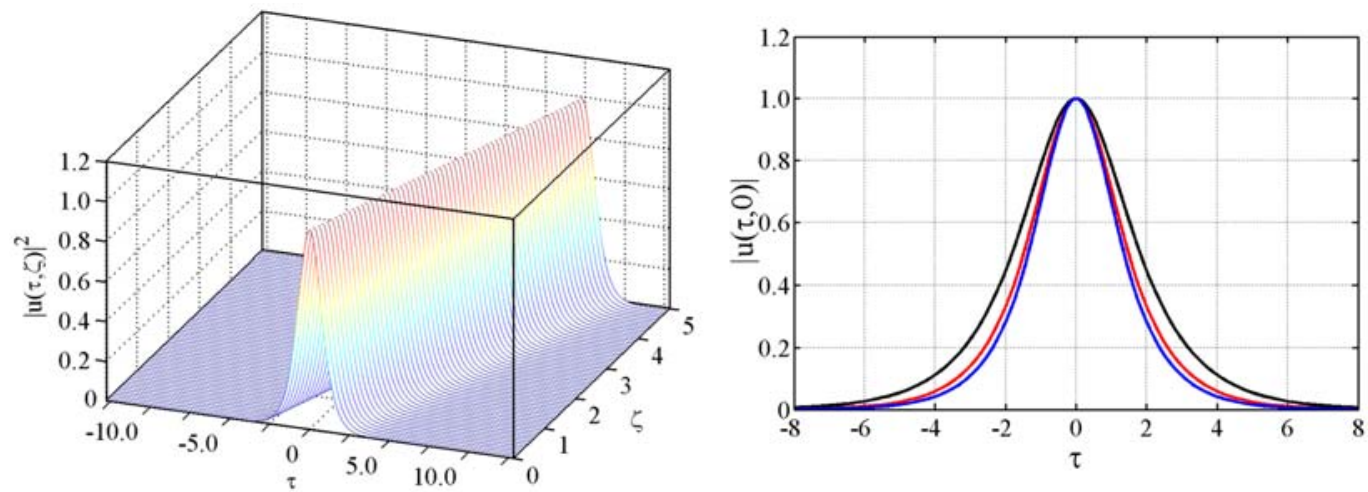

Fig. 1. Left: evolution of an exact bright soliton of model (1) in the $(\tau, \zeta)$ plane. Right: Pulse broadening phenomenon when $\kappa>0$.

Model (1) is a temporal analogue of the spatial nonlinear Helmholtz equation [2]. Hence, one may deploy mathematical and computational techniques that are similar to those used over recent years to analyse broad scalar nonlinear beams (see Fig. 1). We have derived exact analytical bright and dark solitons of Eq. (1). The geometry of these new pulse solutions, which complement their spatial counterparts [3], has been explored in detail. They exhibit generic features (for instance, one encounters both forward- and backward-propagating solution families), and map directly onto a Lorentz-type transformation. More specifically, we have discovered that the velocity combination rule for Helmholtz soliton pulses is strongly reminiscent of that encountered in relativistic particle 
mechanics. Further analytical work has led to the derivation of new invariance laws and conserved quantities. Importantly, the predictions of conventional pulse theory can be recovered in an appropriate simultaneous multiple limit.

Recent computations, in conjunction with linear analysis and nonlinear stability criteria, have predicted that the soliton pulses of Eq. (1) tend to be robust against perturbations to their temporal shape. This key result provides compelling evidence for the stability of Helmholtz pulses in generic dispersive nonlinear systems.

\section{Soliton stability}

In conventional modelling, the well-known Vakhitov-Kolokolov (VK) integral criterion is routinely applied when investigating the stability of bright pulses in NLS-type systems. If $P(\beta)$ is the integrated light intensity and $\beta$ denotes the nonlinear phase shift, one expects the pulse to be stable against small perturbations if the inequality

$$
\frac{\mathrm{d} P}{\mathrm{~d} \beta} \equiv \frac{\mathrm{d}}{\mathrm{d} \beta} \int_{-\infty}^{+\infty} \mathrm{d} \tau|u(\tau, \zeta ; \beta)|^{2}>0
$$

is satisfied. By combining the VK criterion with the space-time geometry of the Helmholtz pulses, we have found that Eq. (2) can also be used to predict the stability of the bright-pulse solutions of Eq. (1). This result is physically intuitive - stability properties must be insensitive to the frame of reference from which one observes the pulse, either the laboratory frame (the Helmholtz model) or a local time frame (conventional models). Previously, we have used similar methods and symmetry arguments to predict the stability of Helmholtz spatial soliton [4].

Computational analyses [5] have shown that conventional pulses of the form $u(\tau, 0)=\eta \operatorname{sech}(\eta \tau) \exp (i \Omega \tau)$ launched into the spatially-dispersive system evolve into exact solitons of Eq. (1). The evolving pulse undergoes self-reshaping oscillations - in the amplitude, width and area - that vanish as $\zeta \rightarrow \infty$ to leave a stationary Helmholtz pulse (see Fig. 2). In this way, Helmholtz solutions may be characterized as robust attractors of the system.
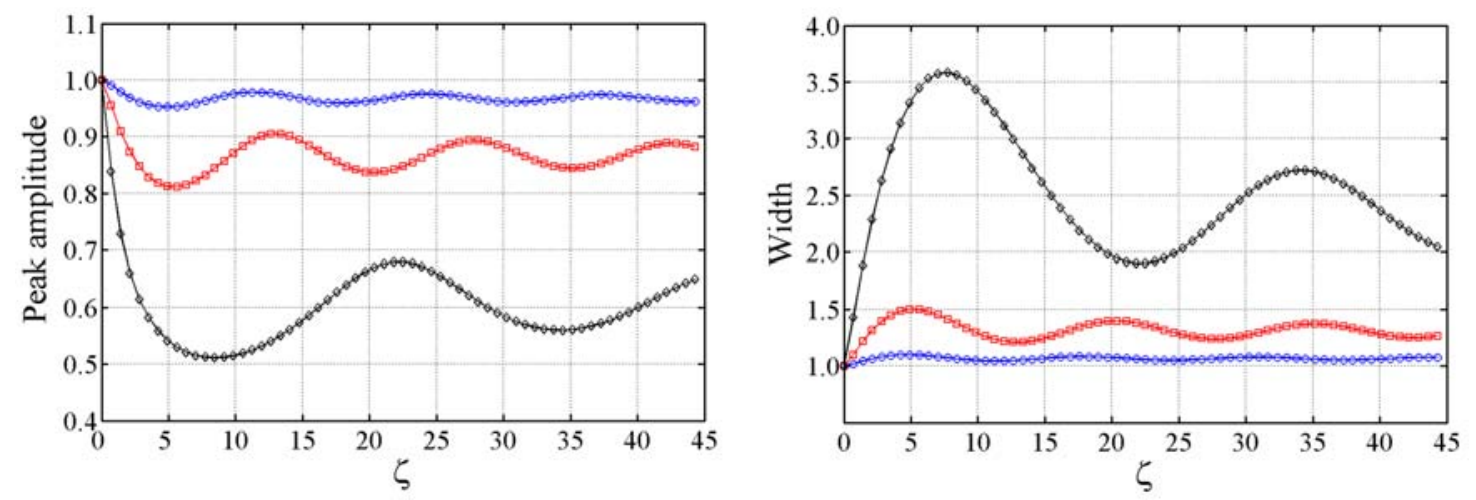

Fig. 2. Evolution of a perturbed input pulse toward a stationary (i.e., exact) Helmholtz soliton as it travels along the $\zeta$ axis of the waveguide. Blue line: weak perturbation; red line: moderate perturbation; black line: strong perturbation.

\section{References}

[1] F. Biancalana F and C.Creatore, "Instabilities and solitons in systems with spatiotemporal dispersion,” Opt. Exp. 16, 14882-93 (2008).

[2] P. Chamorro-Posada, G. S. McDonald, and G. H. C. New, "Non-paraxial solitons,” J. Mod. Opt. 45, 1111-1121 (1998).

[3] P. Chamorro-Posada, G. S. McDonald, and G. H. C. New, "Propagation properties of non-paraxial spatial solitons," J. Mod. Opt. 47, 1877$1886(2000)$.

[4] J. M. Christian , G. S. McDonald, and P. Chamorro-Posada, "Bistable Helmholtz bright solitons in saturable materials," J. Opt. Soc. Am. B 26, 2323-30 (2009).

[5] P. Chamorro-Posada, G. S. McDonald, and G. H. C. New, "Non-paraxial beam propagation methods,” Opt. Commun. 192 1-12 (2000). 